\title{
Encuesta sobre humidificación de la vía aérea en unidades de cuidados intensivos de adultos de Chile
}

\author{
JAIME RETAMAL, JUAN CASTILLO ${ }^{a}$, \\ GUILLERMO BUGEDO, ALEJANDRO BRUHN
}

\section{Airway humidification practices in Chilean intensive care units}

\begin{abstract}
Background: In patients with an artificial airway, inspired gases can be humidified and heated using a passive (heat and moisture exchange filter-HMEF), or an active system (heated humidifier). Aim: To assess how humidification is carried out and what is the usual clinical practice in this field in Chilean intensive care units (ICUs). Material and Methods: A specific survey to evaluate humidification system features as well as caregivers' preferences regarding humidification systems, was carried out on the same day in all Chilean ICUs. Results: Fifty-five ICUs were contacted and 44 of them completed the survey. From a total of 367 patients, 254 (69\%) required humidification because they were breathing through an artificial airway. A heated humidifier was employed only in 12 patients (5\%). Forty-three ICUs (98\%) used HMEF as their routine humidification system. In 52\% of surveyed ICUs, heated humidifiers were not available. Conclusions: In Chile the main method to humidify and heat inspired gases in patients with an artificial airway is the HMEF. Although there are clear indications for the use of heated humidifiers, they are seldom employed.
\end{abstract}

(Rev Med Chile 2012; 140: 1425-1430).

Key words: Intubation, intratracheal; Nebulizers and Vaporizers; Respiration, artificial.

a intubación traqueal elimina los mecanis-
mos naturales de filtración, humidificación
y calentamiento del aire inspirado'. Para
compensar estas funciones, se han desarrollado
sistemas que se instalan en el circuito del ventila-
dor mecánico y permiten entregar el aire humidi-
ficado y temperado a los pulmones
Existen sistemas pasivos y activos. Los sistemas
pasivos son intercambiadores de calor y humedad
que se ubican proximales a la entrada del tubo
traqueal, donde retienen la humedad y el calor
del gas exhalado, y que subsecuentemente entre-
gan esta humedad y calor al gas inspirado. Se les
conoce como HME, del inglés heat and moisture
exchanger, y generalmente están acoplados a un
filtro antibacteriano (HMEF). Los sistemas acti-
vos son calentadores térmicos que se ubican en la

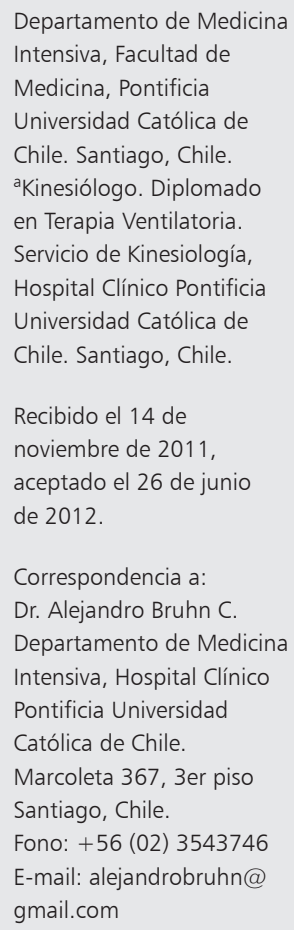

rama inspiratoria del circuito ventilatorio, y que calientan y humidifican el gas inspirado al hacerlo pasar por una cámara de agua temperada donde éste se satura de vapor de agua ${ }^{3}$.

Originalmente se utilizaban sistemas de humidificación activa durante la ventilación mecánica, existiendo una introducción gradual de los sistemas pasivos durante las últimas décadas. Durante los últimos años se han publicado varios estudios que han evaluado y comparado ambos métodos, con resultados variables ${ }^{4-6}$. No se ha demostrado diferencias significativas en la incidencia de neumonía, obstrucción de tubos, o mortalidad al comparar ambos métodos ${ }^{4,5,7}$.

Los HMEF tienen la capacidad de filtrar partículas y microorganismos, y pudiesen, por este mecanismo, disminuir la colonización bacteriana 
de los circuitos del ventilador ${ }^{8}$. Además, tienen un menor costo y representan una menor carga de trabajo de enfermería ${ }^{9,10}$. Sus principales desventajas son que aumentan el espacio muerto instrumental y que se ha reportado una menor capacidad de humidificar y temperar el aire inspirado al comparárseles con sistemas activos y de este modo pudiese significar un aumento en las complicaciones de la vía aérea. Los humidificadores activos son más efectivos para temperar y humidificar el gas inspirado, por lo que pueden asociarse a una menor probabilidad de obstrucción del tubo traqueal por secreciones ${ }^{4,11}$, y adicionalmente, no aumentan el espacio muerto instrumental, permitiendo un mejor manejo del $\mathrm{CO}_{2}^{4,12}$.

Ambos sistemas, activos y pasivos, tienen distintas características físicas y biológicas y por esto, la indicación de uno por sobre el otro debiese ser de acuerdo a las condiciones clínicas de cada enfermo. Tenemos la impresión subjetiva de que en nuestro medio la elección del método de humidificación es por rutina y no está basado en las características individuales de cada paciente.

En Chile no existen datos que indiquen qué métodos de humidificación se utilizan, ni qué criterios son utilizados para realizar esa elección. Por este motivo, decidimos realizar un estudio de prevalencia para conocer qué sistemas de humidificación se usan en las unidades de cuidados intensivos (UCI) de adultos chilenas, y los fundamentos que declaran los clínicos para elegirlos.

\section{Material y Métodos}

Estudio observacional de prevalencia en un día, mediante una encuesta realizada sobre pacientes que se encontraban hospitalizados en UCI, en Chile, el día 7 de diciembre de 2010. El estudio se enfocó en los pacientes que utilizaban ventilación mecánica o que respiraban a través de una vía aérea artificial.

Se incluyeron UCIs de adultos mixtas (médicas y quirúrgicas), excluyéndose a las unidades pediátricas, cardio-quirúrgicas y cardiológicas. Se contactó vía telefónica a los jefes de unidad o enfermeras coordinadoras con el fin de informar los objetivos y pedir colaboración con el estudio. Luego, se envió por e-mail un cuestionario de selección múltiple destinado a conocer las razones para elegir un método u otro, y cuál era la dispo- nibilidad real de cada método en sus unidades. Además se adjuntó una planilla para caracterizar individualmente a los pacientes que se encontraban en la unidad el día de la encuesta, enfocándose en tres variables:

1. Qué tipo de vía aérea tenía el paciente (natural, tubo orotraqueal o traqueostomía),

2. Tipo de soporte ventilatorio (ventilación mecánica invasiva, no invasiva, ventilación espontánea sin presión positiva) y

3. Qué método de humidificación utilizaba el paciente (HMEF o humidificación activa).

Las encuestas remitidas fueron almacenadas y procesadas mediante el programa Google docs. El análisis estadístico se realizó mediante el software Stata 11 (Chicago, EUA). Los datos se presentan como frecuencia (\%) y/o como mediana (rango). La frecuencia de uso de los distintos sistemas de humidificación se comparó mediante análisis de $\square^{2}$ y se consideró como significativa una $\mathrm{p}<0,05$.

\section{Resultados}

Se contactaron cincuenta y cinco UCIs desde Arica hasta Punta Arenas, cuarenta y cuatro de ellas contestaron la encuesta completa, que dan cuenta de 397 camas críticas. Veinte (45\%) unidades correspondían a la Región Metropolitana, y $30(68 \%)$ pertenecían al sistema público. Se registraron 367 pacientes el día del estudio, lo que corresponde a $92 \%$ de ocupación de camas críticas.

En la gran mayoría de las UCIs encuestadas (43/44, 98\%) se utilizaba HMEF como sistema de humidificación de rutina. Las razones que manifestaron los encuestados para preferir un método u otro son mostradas en la Figura 1. La frecuencia típica de recambio de los HMEF reportada es cada $24 \mathrm{~h}$, variando entre $12 \mathrm{~h}$ y una semana (Figura 2). Se comunicaron 10 modelos de HME, con capacidad de humidificación variable $\left(25,8-33,5 \mathrm{mg} \mathrm{H}_{2} \mathrm{O} / \mathrm{L}\right)$ y espacio muerto con una de mediana $58 \mathrm{ml}$ (rango 38-81). Sólo una UCI reportó utilizar humidificación activa como método de rutina. Más de la mitad de las unidades encuestadas $(23 / 44,52 \%)$ no disponen de sistemas de humidificación activa. Entre las 21 unidades que sí disponen de humidificación activa se contabilizaron un total de 86 bases calefactoras, 2 (1-10) equipos por UCI, que corresponde a 1 


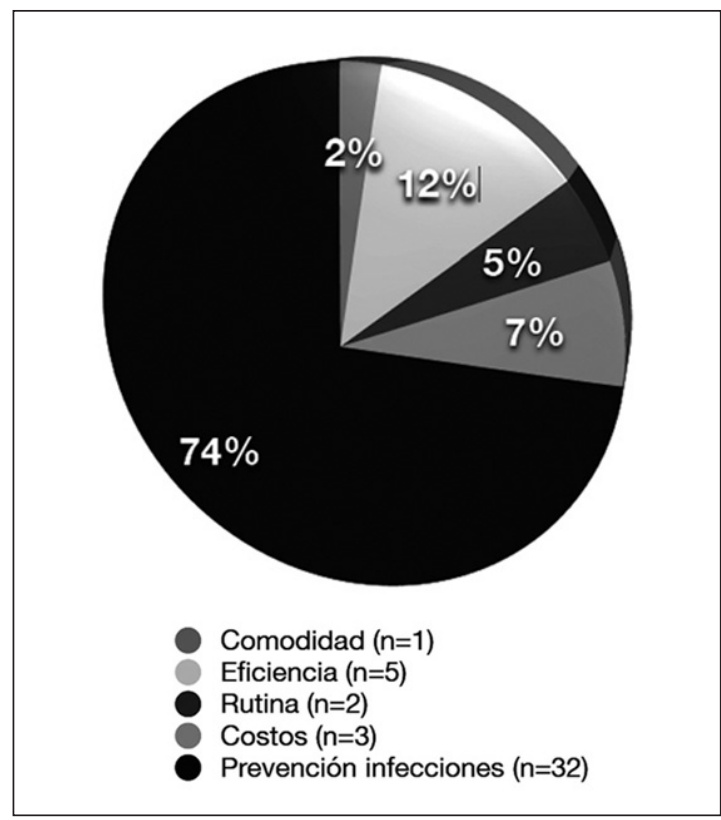

Figura 1.

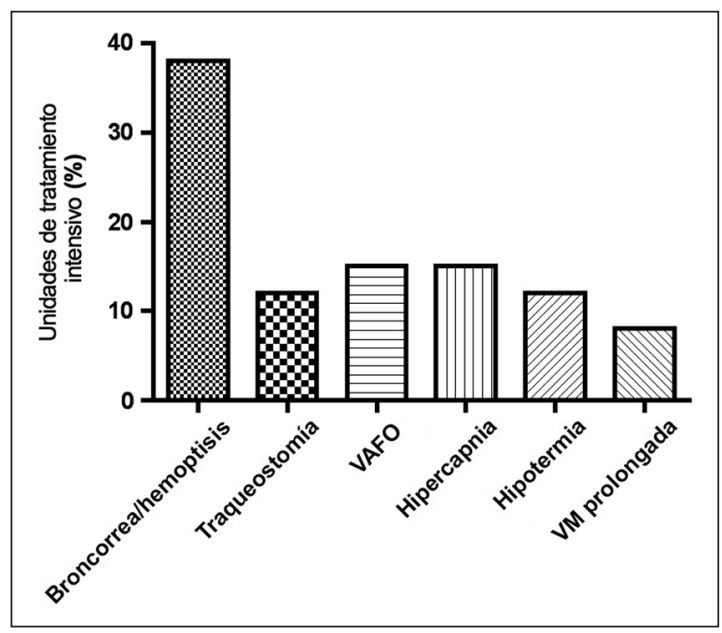

Figura 3.

equipo cada 4,5 camas. El día de la encuesta sólo 12 bases calefactoras se encontraban en uso. Tres UCIs reportaron no utilizar humidificación activa en ninguna circunstancia a pesar de disponer de bases calefactoras. La Figura 3 muestra las indicaciones más frecuentemente reportadas para emplear humidificación activa.

De los 367 pacientes reportados el día de la

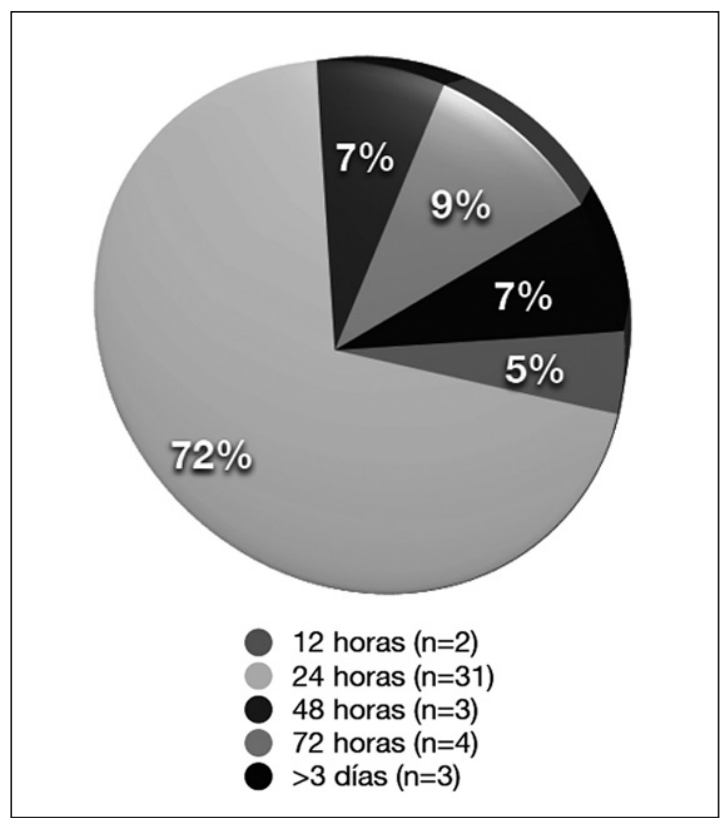

Figura 2.

encuesta, 103 (28\%) se encontraban sin vía aérea artificial ni soporte ventilatorio, $229(62,4 \%)$ estaban con ventilación mecánica invasiva, 25 (6,8\%) con vía aérea artificial pero sin asistencia ventilatoria, y 10 pacientes $(2,7 \%)$ estaban con ventilación mecánica no invasiva. El tipo de humidificación empleado en los pacientes se reporta en la Tabla 1. La gran mayoría de los pacientes conectados a ventilación mecánica invasiva usaban un HMEF $(95,6 \%)$, mientras que sólo 8 pacientes $(3,5 \%)$ usaban humidificación activa. De los 25 pacientes que respiraban espontáneamente a través de vía aérea artificial, 16 (64\%) empleaban el sistema de burbujeo de agua a temperatura ambiente, el mismo que se emplea en la oxígenoterapia habitual de pacientes que respiran a través de su vía aérea natural.

\section{Discusión}

En el presente estudio sobre sistemas de humidificación observamos que los HMEF se utilizan como primera opción en casi la totalidad de los centros encuestados, principalmente por razones de "seguridad infectológica" y menor costo. Los sistemas de humidificación activa se utilizan 
Tabla 1. Uso de distintos tipos de humidificación según ventilación y vía aérea

\begin{tabular}{|lccccc|}
\hline & \multicolumn{5}{c|}{ Tipo de humidificador } \\
Tipo de vía aérea y ventilación & $\mathbf{n}$ & HMEF & HA & Burbuja & p \\
Traqueostomía & 46 & 31 & 5 & 10 & $<0,05$ \\
Asistencia ventilatoria & 29 & 24 & 3 & 2 & 8 \\
Ventilación espontánea & 17 & 7 & 2 & 8 & $<0,05$ \\
Tubo endotraqueal & 208 & 195 & 5 & 0 & $<0,05$ \\
Asistencia ventilatoria & 200 & 195 & 5 & 0 & $<0,05$ \\
Ventilación espontánea & 8 & 0 & 0 & & 8 \\
\hline
\end{tabular}

HMEF: filtro intercambiador de humedad; HA: humidificación activa.

excepcionalmente y están disponibles sólo en algunos centros.

Los pacientes que ventilan a través de una vía aérea artificial requieren de algún sistema de humidificación que asegure condiciones de temperatura y humedad óptimas al gas inspirado que llega a la vía aérea. Para ello pueden emplearse humidificadores activos o HMEF. De 254 pacientes que se encontraban con vía aérea artificial, 236 estaban utilizando alguna de estas dos opciones. Sin embargo, en 18 pacientes se estaba aplicando humidificación por burbujeo de agua a temperatura ambiente. Interesantemente, estos pacientes se encontraban en ventilación espontánea. La humidificación por burbujeo de agua a temperatura ambiente no provee condiciones aceptables de humedad y temperatura en pacientes con vía aérea artificial $^{13}$, independiente de si se encuentran en ventilación espontánea o asistida. Estos datos reflejan, quizás, un desconocimiento respecto de las indicaciones y de la racionalidad del uso de sistemas de humidificación en los pacientes críticos. El uso de sistemas de humidificación en los pacientes intubados o traqueostomizados se justifica por la pérdida de la función acondicionadora de la vía aérea superior, más que por el hecho de que se esté empleando o no ventilación mecánica.

No existe consenso respecto al nivel óptimo de humedad y temperatura que se debe alcanzar en los pacientes críticos, aunque las recomendaciones de temperatura varían entre 29 y $32^{\circ} \mathrm{C}$, y la humedad relativa entre 95 y 100\%. Estas condiciones pueden obtenerse mediante un sistema activo, y en algunas circunstancias también pueden alcanzarse con HMEF, siendo los sistemas activos en general más eficaces. Tanto los sistemas activos como los HMEF tienen sus propias limitaciones. Aunque la tendencia en las últimas décadas ha sido utilizar de preferencia HMEF como primera opción, existen frecuentemente condiciones en que su uso está contraindicado, o bien en que existe una clara ventaja a favor de los sistemas activos. Por ejemplo, destete difícil en pacientes con falla respiratoria crónica ${ }^{14}$, ventilación oscilatoria de alta frecuencia ${ }^{15}$, hipotermia, hemoptisis, broncorrea, ventilación mecánica con bajo volumen corriente ${ }^{16}$ y fístula broncopleura ${ }^{17}$. En este sentido, llama la atención la baja tasa de uso de sistemas de humidificación activa observada en este estudio $(<5 \%)$. Más aun, resulta relevante que en prácticamente la mitad de las unidades del país no se dispone de estos sistemas. Sin embargo, ésta no parece ser la razón fundamental que explica su escaso uso ya que aun en las unidades que sí cuentan con bases calefactoras el uso de humidificación activa no supera el $10 \%$. Nuestra realidad contrasta fuertemente con un estudio similar realizado en Francia y Canadá. En Francia, país en donde se prefiere HMEF como primera opción, los sistemas activos son usados por más de $24 \%$ de los pacientes. En Canadá en cambio, la opción de preferencia es la humidificación activa, cuyo uso supera el $60 \%{ }^{18}$.

El uso de HMEFs se ha asociado a menores $\operatorname{costos}^{4,17,19}$. En general, los fabricantes de HMEF sugieren su reemplazo por uno nuevo cada $24 \mathrm{~h}$, $o$ antes si se contaminan con secreciones o se dañan. Sin embargo, la evidencia y las guías clínicas sugieren que su recambio puede realizarse cada 48 a 96 h, incluso hasta cada una semana, sin existir aumento en la incidencia de neumonía, reducción 
en la capacidad de humidificación, ni tampoco aumento de la resistencia de la vía aérea ${ }^{10,19-21}$. Según los resultados de la encuesta, la mayoría de las UCIs recambian el HMEF cada $24 \mathrm{~h}$. La decisión de modificar esta conducta permitiría reducir costos innecesarios y los riesgos asociados a la apertura del circuito. Destaca el gran espectro de marcas de HMEF usados el día de la encuesta, la gran mayoría de ellas con una adecuada eficiencia de humidificación reportada ( $\left.>30 \mathrm{mgH}_{2} \mathrm{O} / \mathrm{L}\right)$. Sin embargo, Lellouche et al demostraron diferencias significativas entre la eficiencia de humidificación publicada por los fabricantes y los medidos en laboratorio ${ }^{22}$.

Diversos estudios y metaanálisis han relacionado el uso de los diferentes sistemas de humidificación a la incidencia de neumonía asociada a la ventilación mecánica (NAVM). Sin embargo, existen datos contradictorios, lo cual no permite generar una recomendación clara al respecto ${ }^{4,5}$. A favor del uso de HMEFs podemos afirmar que permiten mantener el circuito del ventilador cerrado y que tienen un filtro viral y bacteriano asociado. Los humidificadores activos se han asociado a una mayor incidencia de colonización de los circuitos, sin embargo, no se ha demostrado que esta condición se asocie a un aumento en la incidencia de $\mathrm{NAVM}^{8,23}$. Además, la acumulación de agua en los circuitos se ha reducido mediante la inclusión de alambres calefactores y al desarrollo de circuitos permeables al vapor de agua.

Este estudio constituye el primer reporte de datos de humidificación en Chile y aporta datos importantes y representativos para la toma de decisiones. Sin embargo, reconocemos que presenta limitaciones importantes ya que por el carácter descriptivo del estudio, no se exploraron las complicaciones asociadas al uso de sistemas de humidificación (NAVM, obstrucción de tubo endotraqueal y mortalidad).

Podemos concluir que en nuestro país el método preferido para humidificar y temperar el aire inspirado es HMEF. El uso de humidificación activa es extremadamente bajo en relación a datos publicados en otros países. Es muy llamativo que varios hospitales no cuentan con sistemas de humidificación activa considerando que existen pacientes con contraindicaciones para el uso de HMEFs (ej: broncorrea importante, hemoptisis u obstrucción bronquial severa). El desarrollo de guías y normas de humidificación locales podría permitir una elección más apropiada e individualizada de los métodos de humidificación disponibles.

\section{Referencias}

1. Bench S. Humidification in the long-term ventilated patient; a systematic review. Intensive Crit Care Nurs 2003; 19 (2): 75-84.

2. Cinnella G, Giardina C, Fischetti A, Lecce G, Fiore MG, Serio G, et al. Airways humidification during mechanical ventilation. Effects on tracheobronchial ciliated cells morphology. Minerva Anestesiol 2005; 71 (10): 585-93.

3. Chiumello D, Pelosi P, Park G, Candiani A, Bottino N, Storelli E, et al. In vitro and in vivo evaluation of a new active heat moisture exchanger. Crit Care 2004; 8 (5): R281-8.

4. Kelly M, Gillies D, Todd DA, Lockwood C. Heated humidification versus heat and moisture exchangers for ventilated adults and children. Cochrane Database Syst Rev (4): CD004711.

5. Kola A, Eckmanns T, Gastmeier P. Efficacy of heat and moisture exchangers in preventing ventilator-associated pneumonia: meta-analysis of randomized controlled trials. Intensive Care Med 2005; 31 (1): 5-11.

6. Siempos, II, Vardakas KZ, Kopterides P, Falagas ME. Impact of passive humidification on clinical outcomes of mechanically ventilated patients: A meta-analysis of randomized controlled trials ${ }^{*}$. Crit Care Med 2007.

7. Lacherade JC, Auburtin M, Cerf C, Van de Louw A, Soufir L, Rebufat Y, et al. Impact of humidification systems on ventilator-associated pneumonia: a randomized multicenter trial. Am J Respir Crit Care Med 2005; 172 (10): 1276-82.

8. Dreyfuss D, Djedaini K, Gros I, Mier L, Le Bourdelles G, Cohen Y, et al. Mechanical ventilation with heated humidifiers or heat and moisture exchangers: effects on patient colonization and incidence of nosocomial pneumonia. Am J Respir Crit Care Med 1995; 151 (4): 986-92.

9. Boyer A, Thiery G, Lasry S, Pigne E, Salah A, de Lassence A, et al. Long-term mechanical ventilation with hygroscopic heat and moisture exchangers used for 48 hours: a prospective clinical, hygrometric, and bacteriologic study. Crit Care Med 2003; 31 (3): 823-9.

10. Thomachot L, Leone M, Razzouk K, Antonini F, Vialet $\mathrm{R}$, Martin C. Randomized clinical trial of extended use of a hydrophobic condenser humidifier: 1 vs 7 days. Crit Care Med 2002; 30 (1): 232-7.

11. Cohen IL, Weinberg PF, Fein IA, Rowinski GS. Endotracheal tube occlusion associated with the use of heat and 
moisture exchangers in the intensive care unit. Crit Care Med 1988; 16 (3): 277-9.

12. Prat G, Renault A, Tonnelier JM, Goetghebeur D, Oger E, Boles JM, et al. Influence of the humidification device during acute respiratory distress syndrome. Intensive Care Med 2003; 29 (12): 2211-5.

13. Chanques G, Constantin JM, Sauter M, Jung B, Sebbane $\mathrm{M}$, Verzilli $\mathrm{D}$, et al. Discomfort associated with underhumidified high-flow oxygen therapy in critically ill patients. Intensive Care Med 2009; 35 (6): 996-1003.

14. Girault C, Breton L, Richard JC, Tamion F, Vandelet P, Aboab J, et al. Mechanical effects of airway humidification devices in difficult to wean patients. Crit Care Med 2003; 31 (5): 1306-11.

15. Fessler HE, Derdak S, Ferguson ND, Hager DN, Kacmarek RM, Thompson BT, et al. A protocol for highfrequency oscillatory ventilation in adults: results from a roundtable discussion. Crit Care Med 2007; 35 (7) 1649-54.

16. Branson RD. Humidification of respired gases during mechanical ventilation: mechanical considerations. Respir Care Clin N Am 2006; 12 (2): 253-61.

17. Branson RD. The ventilator circuit and ventilatorassociated pneumonia. Respir Care 2005; 50 (6): 774-85; discussion 785-7.
18. Ricard JD, Cook D, Griffith L, Brochard L, Dreyfuss D. Physicians' attitude to use heat and moisture exchangers or heated humidifiers: a Franco-Canadian survey. Intensive Care Med 2002; 28 (6): 719-25.

19. Kollef MH, Shapiro SD, Boyd V, Silver P, Von Harz B, Trovillion E, et al. A randomized clinical trial comparing an extended-use hygroscopic condenser humidifier with heated-water humidification in mechanically ventilated patients. Chest 1998; 113 (3): 759-67.

20. Ricard JD, Le Miere E, Markowicz P, Lasry S, Saumon G, Djedaini K, et al. Efficiency and safety of mechanical ventilation with a heat and moisture exchanger changed only once a week. Am J Respir Crit Care Med 2000; 161 (1): 104-9.

21. AARC clinical practice guideline. Humidification during mechanical ventilation. American Association for Respiratory Care. Respir Care 1992; 37 (8): 887-90.

22. Lellouche F, Taille S, Lefrancois F, Deye N, Maggiore SM, Jouvet $\mathrm{P}$, et al. Humidification performance of 48 passive airway humidifiers: comparison with manufacturer data. Chest 2009; 135 (2): 276-86.

23. Craven DE, Goularte TA, Make BJ. Contaminated condensate in mechanical ventilator circuits. A risk factor for nosocomial pneumonia? Am Rev Respir Dis 1984; 129 (4): 625-8. 